\title{
Redundant visual signals boost saccade execution
}

\author{
MASSIMO TURATTO and ELENA BETTA \\ University of Trento, Rovereto, Italy
}

\begin{abstract}
The redundant signal effect (RSE) refers to the fact that human beings react more quickly to a pair of stimuli than to only one stimulus. In previous studies of the RSE in the oculomotor system, bimodal signals have been used as the goal of the saccade. In consistency with studies using manual response times (RTs), saccadic RTs have been shown to be shorter for redundant multimodal stimuli than for single unimodal stimuli. In the present experiments, we extended these findings by demonstrating an RSE in the saccadic system elicited only by unimodal visual stimuli. In addition, we found that shorter saccadic RTs were accompanied by an increased saccadic peak velocity. The present results are of relevance for neurophysiological models of saccade execution, since the boost of saccades was elicited by two visual transients (acting as a "go" signal) that were presented not at the goal of the saccade but at various other locations.
\end{abstract}

When required to respond manually as quickly as possible to a stimulus onset, the human being delivers a faster response when two stimuli are presented than when only one is presented. This phenomenon is known as the redundant signal effect (RSE; Todd, 1912). The response time (RT) advantage observed in the double-signal over the single-signal condition is also called redundancy gain, and has been documented for stimuli delivered in the same mode and in different modalities (see, e.g., Miller, 1982, 1986; Mordkoff \& Yantis, 1991). The redundancy gain can be explained according to either statistical facilitation (Raab, 1962) or neural coactivation (Miller, 1982), with the former postulating a horse race between stimuli for response activation and the latter hypothesizing that stimuli are pooled together before the response is emitted. To distinguish between these two possibilities, Miller (1982) devised a mathematical method known as the horse-race inequality. If the inequality is not violated, probability summation is sufficient to explain the redundancy gain; in contrast, if a violation is observed, some sort of neural coactivation should be assumed to have occurred.

Although most of the RSE studies have recorded manual RTs (Miller \& Reynolds, 2003), a few have addressed the RSE using saccadic responses (Arndt \& Colonius, 2003; Colonius \& Arndt, 2001; Hughes, Reuter-Lorenz, Nozawa, \& Fendrich, 1994). Notably, whereas manual RT studies have demonstrated the redundancy gain for both visual and multimodal stimuli, in those in which saccadic RTs were recorded only multimodal stimuli were used. Typically, in the latter studies the redundant signal con-

The authors thank Anne Hillstrom, John Findlay, and two anonymous reviewers for their very helpful comments on an early draft of the manuscript. Thanks are also due Sabrina Cantatore for her help in data collection. Correspondence concerning this article should be addressed to M. Turatto, Dipartimento di Scienze della Cognizione e della Formazione, Via Matteo del Ben, 5, 38068 Rovereto, Italy (e-mail: massimo.turatto@unitn.it). dition consists of auditory and visual stimuli presented together at the same location (corresponding to the saccadic goal), whereas the single-signal condition consists of a unimodal stimulus. The results consistently showed shorter saccadic latencies on double- than on single-signal trials (see, e.g., Hughes et al., 1994).

Quite surprisingly, what has remained unexplored is whether or not redundant unimodal visual stimuli can produce an RSE in saccadic RTs. Hence, the aim of the present study was to address whether or not the execution of a visually guided endogenous saccade toward a prespecified target can be speeded up by a "go" signal consisting of two visual transients instead of only one, with the visual transients appearing at nontarget locations.

\section{EXPERIMENT 1}

The only evidence that unimodal visual stimuli can elicit an RSE comes from studies in which manual RTs were recorded (see, e.g., Miller, 2004; Miller \& Reynolds, 2003). In these studies, the participants' task was to press a button as soon as they detected the occurrence of a visual transient ("go" signal). Note that the motor response in this task is not directed toward the "go" signal. Hence, to determine whether or not unimodal visual stimuli can elicit an RSE in the oculomotor system, we presented visual transients that did not coincide with the saccadic goal. In other words, our aim was to study the RSE in visually guided endogenous saccades that were not directed toward the "go" signal, rather than in exogenous saccades.

Furthermore, by dissociating the locations of the saccadic target and the visual transients, results from Experiments 1 and 2 can be related (see the General Discussion section) to those from recent studies investigating the distracting effect of visual transients on the programming of endogenous saccades toward a no-onset target (Godijn \& Theeuwes, 2002; Kramer, Cassavaugh, Irwin, \& Peterson, 2001). 


\section{Method}

Participants. Ten students ( 7 females) from the University of Trento (age range, 20-26 years) voluntarily took part in Experiment 1 . All were naive as to the purpose of the study, had normal or corrected-to-normal vision, and gave informed written consent to participate in the study.

Apparatus, Stimuli, and Procedure. Each participant sat with his or her head on a chinrest at approximately $72 \mathrm{~cm}$ in front of a Dell CRT 19-in. $(1,024 \times 768$ pixel, $75-\mathrm{Hz})$ monitor. Generation and presentation of the stimuli were controlled by a custom-made C program running under Windows 2000 on a Pentium IV Dell PC. Eye movements were recorded by an Eyelink II system (SR Research, Ontario, Canada) with a sampling rate of $250 \mathrm{~Hz}$ and a spatial resolution of less than $0.5^{\circ}$ of visual angle. A standard 9-point grid calibration was performed at the beginning of the experiment.

Each trial began with the appearance of two red spots $\left(0.53^{\circ}\right.$ in diameter), one at the center of the screen (the fixation point) and one $5^{\circ}$ above it (the saccadic goal; see Figure 1). The participants had to maintain their gaze within $1^{\circ}$ of the center of the screen for $500 \mathrm{msec}$ in order to trigger drift correction (i.e., detection of the offset of gaze position from fixation position; this offset is used to correct the trial gaze data). A randomly variable time interval (700-1,200 msec) followed, after which either one or two black disks covering $1^{\circ}$ (the "go" signal) were presented for $100 \mathrm{msec}$. The two possible locations for the disks were $5^{\circ}$ to the left and $5^{\circ}$ to the right of fixation, so single-disk signals were presented on one side and double-disk signals were presented bilaterally. The participants were instructed to respond as quickly as possible to the "go" signal by moving their gaze to the red spot above the fixation point. A correct saccadic response was achieved if the first saccade landed in a window of $4^{\circ}$ centered on target location and was signaled by the target spot's becoming green for $500 \mathrm{msec}$. A warning tone sounded if (1) a saccade was made before signal onset, (2) the endpoint of the saccade was outside the target window, or (3) no response was given within $1,500 \mathrm{msec}$ after the "go" signal appeared. The intertrial interval was $1,500 \mathrm{msec}$.

Each participant performed 20 practice and 120 experimental trials. Twenty-four $(20 \%)$ of the latter were catch trials, in which no signal was presented; 48 were double- and 48 single-signal trials, with the latter appearing equiprobably left or right of fixation.

Catch, single-signal, and double-signal trials were presented in random order. Saccadic latency (i.e., the time it took the participant to initiate the saccade) was measured from the onset of the "go" signal.

\section{Results}

Saccadic latency. The participants made a saccade in $4 \%$ of the catch trials. On regular trials, $3 \%$ of the responses were excluded from analysis because of a missing response (1) or fixation errors (28). In this and the following experiment, no saccadic RTs shorter than $150 \mathrm{msec}$ were found, and those above $1,000 \mathrm{msec}(0.35 \%$ of the trials in Experiment 1) were trimmed before data analysis. As can be seen in Table 1, the participants showed shorter saccadic latency on double-signal $(M=313 \mathrm{msec}, S D=$ 88 ) than on single-signal $(M=349 \mathrm{msec}, S D=105)$ trials $[t(9)=5.215, p<.001 ;$ all reported $t$ tests are two-tailed $]$.

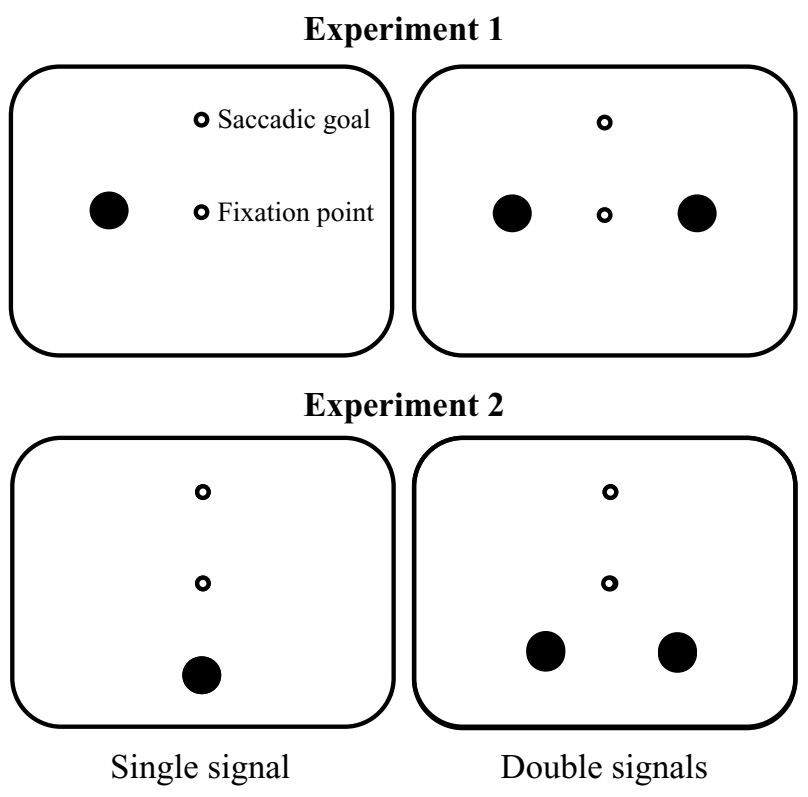

Figure 1. Single-signal (left) and double-signal (right) conditions in Experiments 1 (top) and 2 (bottom). Stimuli are not drawn to scale.

RTs to left $(M=352 \mathrm{msec})$ and right $(M=347 \mathrm{msec})$ signals were not significantly different $(p=.7)$.

Saccadic accuracy. It is possible that saccadic RTs were longer on single- than on double-signal trials because in the former the participants had to inhibit a reflex saccade toward the single peripheral signal, whereas the inhibition of such oculomotor capture was absent or less pronounced for the bilateral signals. To investigate the distracting effect of single and double signals, we analyzed the distribution of inaccurate saccades (i.e., saccades that landed out of the $4^{\circ}$ window centered on target location). On average, $6.75 \%$ of saccades were inaccurate, but the difference between the single-signal $(8.6 \%)$ and doublesignal $(4.9 \%)$ conditions was not significant $(p=.168)$. However, all of the erroneous horizontal saccades (i.e., those landing within a $45^{\circ}$ sector centered along the horizontal meridian and departing from fixation-16 out of 63) were elicited in the single-signal condition. Although the number of these saccades was extremely low, this might suggest a weak tendency for the single signal to capture the eyes.

Saccadic peak velocity. The peak velocity of accurate saccades was also analyzed (see Table 1). Saccades in response to double signals had a higher peak velocity $\left(M=265^{\circ} / \mathrm{sec}, S D=32\right)$ than saccades in response to

Table 1

Saccadic Latency and Peak Velocity in Experiments 1 and 2

\begin{tabular}{cccccc}
\hline & \multicolumn{2}{c}{ Latency $(\mathrm{msec})$} & & \multicolumn{2}{c}{ Peak Velocity $(\mathrm{deg} / \mathrm{sec})$} \\
\cline { 2 - 3 } \cline { 5 - 6 } Experiment & Single Signal & Double Signals & & Single Signal & Double Signals \\
\hline 1 & 349 & 313 & & 255 & 265 \\
2 & 334 & 316 & & 259 & 268 \\
\hline
\end{tabular}


single signals $\left(M=255^{\circ} / \mathrm{sec}, S D=26\right)[t(9)=-2.26$, $p<.05]$. Peak velocity declined slightly with increasing latency $(r=-.34$, slope $=-0.13)$. Within-participants slope analysis confirmed a significant decrease in peak velocity with increasing saccadic latency (mean slope $=$ $-0.09, S D=0.12)[t(9)=-2.38, p<.05]$.

Test of the race model. To assess whether the observed redundancy gain was consistent with statistical facilitation or with neural coactivation, we applied the horse-race inequality test (Miller, 1986). For each participant, the cumulative distribution functions (CDFs) of RTs for left and right single signals were computed with a procedure that linearly interpolates the percentile rank in 1-msec bins between the observed RTs. Then, quantiles of the sum of these two CDFs were computed in 40 steps of 0.025 and compared with the corresponding quantiles of the double-signal CDF. A significant violation ( $t$ test, $p<.05$ ) of the inequality was found in the quantiles 0.05 , 0.075 , and 0.1 .

\section{Discussion}

The present results showed that an RSE in the oculomotor system can be obtained by redundant unimodal visual signals, even when the signals are not the goal of the visually guided endogenous saccades (cf. Hughes et al., 1994). Moreover, in addition to reducing saccadic latency, the redundant transients increased saccadic peak velocity. This, together with the violation of the horse-race inequality, suggests that the redundancy gain in saccadic RTs was probably due to neural coactivation at the motor level rather than to statistical facilitation. (Giray \& Ulrich, 1993, reported analogous findings by showing that manual responses to redundant stimuli were faster and more forceful.) These results are in line with the hypothesis that the RSE could be attributable, at least partially, to coactivation at the motor output stage (Miller, 2004).

Moreover, since the RSE was elicited by visual stimuli appearing at locations different from that of the saccadic goal, the mechanism responsible for the saccadic latency reduction would be different from that hypothesized to govern the RSE (by means of multimodal neural integration) for spatially coincident audiovisual stimuli that correspond to the goal of the saccade (see, e.g., Hughes et al., 1994). In the General Discussion section, we explore this issue in more detail.

\section{EXPERIMENT 2}

To rule out the effect of any possible imbalance in the oculomotor capture between single and double signals as the cause of the reduced saccadic latency observed in the redundant condition of Experiment 1, the stimuli in the single- and double-signal conditions were all presented below the fixation point (see Figure 1). Because the stimuli were arranged in this manner, it seems unlikely that the single signal would have exerted a stronger oculomotor capture than the double signals (thus possibly increasing saccadic latency). Indeed, in both conditions the stimulus vector was opposite that of the required saccade and pointed toward the center of gravity between the two signals in the redundant condition (Findlay, 1997).

\section{Method}

Ten students ( 7 females) from the University of Trento (age range, 21-34 years) participated in Experiment 2. None of them had taken part in Experiment 1. The experimental setup and procedure were as in Experiment 1, with the exception of the position of single and double signals. The black disks were always presented $5^{\circ}$ away from the fixation point. However, single signals appeared vertically below the fixation point, whereas double signals appeared in directions forming a $45^{\circ}$ angle with the vertical meridian (see Figure 1).

\section{Results}

Saccadic latency. The participants made saccades in $11 \%$ of the catch trials. On regular trials, $3 \%$ of the responses were excluded from analysis because of fixation errors. The outlier latency criterion removed $0.12 \%$ of the correct trials. As in Experiment 1, the participants showed shorter saccadic latency on double-signal $(M=316 \mathrm{msec}$, $S D=85)$ than on single-signal $(M=334 \mathrm{msec}, S D=99)$ trials $[t(9)=2.889, p<.02$; see Table 1$]$.

Saccadic accuracy. On average, $10.8 \%$ of saccadic responses were inaccurate (i.e., landed out of the $4^{\circ}$ window centered on target location). There was no significant difference in proportion of accurate saccades between conditions ( $11.6 \%$ on average), and only one saccade went below the horizontal meridian. Saccades in response to double signals were slightly but significantly longer $(M=$ $\left.5.10^{\circ}, S D=0.60\right)$ than saccades in response to single signals $\left(M=4.96^{\circ}, S D=0.45\right)[t(9)=2.48, p<.05]$.

Saccadic peak velocity. Accurate saccades had a higher peak velocity in response to double $\left(M=268^{\circ} / \mathrm{sec}\right.$, $S D=41)$ than to single $\left(M=259^{\circ} / \mathrm{sec}, S D=38\right)$ signals $[t(9)=-2.76, p<.03$; see Table 1]. Peak velocity declined slightly with increasing latency $(r=-.1$, slope $=$ $-0.05)$, as was confirmed by within-participants slope analysis [mean slope $=-0.08, S D=0.12 ; t(9)=-2.35$, $p<.05]$.

Test of the race model. No violations of the horserace inequality emerged in the present experiment.

\section{Discussion}

The main findings that emerged in Experiment 1 were replicated even when any possible difference in oculomotor capture between the single- and double-signal conditions was reasonably ruled out. Also, the enhancement in saccadic execution was evident even though, in the double-signal condition, the two stimuli could potentially trigger a reflexive saccade in the direction opposite that of the target.

Unlike in Experiment 1, no violation of the horse-race inequality emerged in the present experiment. This is not inconsistent, however, with the neural coactivation hypothesis (Miller, 1982). The lack of violation might be related to the fact that the visual signals in the double-signal condition were closer to the vertical meridian than were those in Experiment 1. If the superior colliculus (SC) is one of the neural structures in which neural coactivation might take place (Corballis, 1998), stimuli closer to the verti- 
cal meridian could have reduced the possibility of neural coactivation as the $\mathrm{SC}$ receives stronger input from nasal than from temporal hemiretinas (Dodds, Machado, Rafal, \& Ro, 2002; Itaya \& Van Hoesen, 1983). However, although a violation of the horse-race inequality was not found, in our view the increased saccadic peak velocity in the redundant condition is inconsistent with the horse-race model, which can explain a reduction in saccadic RTs but cannot easily accommodate a boost in saccadic peak velocity.

\section{GENERAL DISCUSSION}

This study showed that saccadic latency for a visual target was reduced when two visual transients were used as a "go" signal instead of only one, and this was true even when the transients did not coincide with the saccadic goal. These results extend previous findings, in which a reduction of saccadic latency was documented for a redundant condition in which two stimuli of different modalities appeared at the same location and served as the target of the saccade (see, e.g., Arndt \& Colonius, 2003). Moreover, the present findings show that in the redundant condition the saccadic latency reduction was accompanied by an increased saccadic peak velocity. Such a relation between saccadic latency and peak velocity has been documented in both humans (Ramchandran et al., 2004) and nonhuman primates (Edelman \& Goldberg, 2001).

The task adopted in Experiment 2 resembles somewhat the antisaccade task (Munoz \& Everling, 2004), since in both cases participants are required to make a saccade away from the visual transient(s) occurring on the screen. Paradoxically, from this point of view the results of Experiment 2 would seem to indicate that antisaccades could be faster when two peripheral stimuli are presented instead of only one. However, although in both these tasks the eyes must be directed away from the visual transient(s), an important difference between them is that in our task the saccade vector could be programmed in advance. In contrast, in the antisaccade task the direction of the saccade must be defined (online) on the basis of the spatial coordinates of the visual transient, whereas in Experiment 2 the spatial properties of the visual transients were task irrelevant for initiating the saccade. The impossibility of knowing the target location before the distractor's occurrence would also explain why, in the remote distractor effect, visual transients appearing at a nontarget location increase rather than decrease saccadic RTs to a visual target (Walker, Deubel, Schneider, \& Findlay, 1997). In addition, by having the target saccade already programmed at the time of the visual transients' presentation, in the present study the participants were able to counteract the reflexive oculomotor capture toward the "go" signal. In contrast, when the saccadic goal cannot be specified before the occurrence of the visual transient, as in the standard antisaccade task, the rate of erroneous saccades directed toward this sudden event increases considerably (Munoz \& Everling, 2004).

Recently, Godijn and Theeuwes (2002) showed that saccadic RTs to a no-onset target were longer when a visual transient was simultaneously added to the display than when it was absent (interestingly, this effect does not seem to vary as a function of the number of distracting transients-Kramer et al., 2001). This result, however, is not inconsistent with the present findings. Indeed, since in Godijn and Theeuwes's study the location of the color singleton target was not known in advance, the endogenous programming of the saccade toward it occurred simultaneously and competed with the exogenous programming of the saccade elicited by the onset of the distractor at different locations. By contrast, the saccadic boost caused by visual transients that we documented seems to take place when the endogenous saccade can be programmed in advance, since the target is already visible at the time of presentation of the visual transients.

In our view, the present findings might also relate to the model for saccade generation proposed by Godijn and Theeuwes (2002). Some of the key assumptions made by their competitive integration model are that (1) endogenous and exogenous signals and the corresponding saccade programs are integrated in a common saccade map; (2) when two distant locations on the map are activated, they mutually inhibit each other; (3) fixation-related activity is critical for triggering saccade initiation, inhibiting activations at distant locations, and vice versa; and (4) when a saccade is required, the fixation activity has to be inhibited below the activation threshold.

In regard to the last point, one might also hypothesize that the faster the fixation is inhibited, the shorter the saccadic latency will be. It is therefore conceivable that because in our paradigm the participants had to maintain their eyes at fixation until the visual transient(s) occurred (although the target was visible in advance), a stronger map activity took place at fixation than at the target location. Then, when the visual transient(s) occurred, according to the Godijn and Theeuwes (2002) model an inhibitory signal was sent from the transients' location to both the fixation and the target locations. If one had to consider only the mutual inhibitory activity between visual transients and the target location, our results would be paradoxical, since the target location should turn out to be more strongly inhibited by two visual transients than by only one. This, of course, should have caused longer saccadic RTs in the double- than in the single-signal condition, whereas we obtained the opposite pattern. However, our results can be accommodated in the competitive integration model by a further assumption - namely, that the strength of inhibition spreading from activated locations follows a gradient pattern. If this were the case, upon presentation of visual transients the inhibition would be stronger at fixation than at the target location, the former being closer to the "go" signals than the latter. Hence, the fact that the saccadic latency is directly related to the speed of disengagement from fixation could explain why we observed shorter saccadic RTs with two visual transients than with only one. Indeed, activations in the saliency map caused by the presentation of two "go" signals inhibited the fixation-related activity to a greater extent, leading to a faster disengagement (see below for a possible physiological basis of this effect).

Furthermore, a particularly new finding of Experiment 2 is the saccadic boost caused by redundant visual 
transients presented in two locations opposite that of the saccadic target. To the best of our knowledge, previous evidence of reduced saccadic RTs and increased peak velocity has been observed only in tasks in which the visual transient occurred at the saccadic goal (Edelman \& Goldberg, 2001; Ramchandran et al., 2004). In light of the positive correlation between neural activity in the SC and prosaccade peak velocity, and because the same SC neural activity is negatively correlated with saccadic latency (Dorris, Paré, \& Munoz, 1997), it has been suggested that shorter saccadic latency and higher saccadic peak velocity are due to increased neural activity in the SC when a visual transient occurs at the saccadic goal than when no visual transient is presented (Edelman \& Goldberg, 2001; Ramchandran et al., 2004). However, the present findings showed that a similar enhancement of saccadic execution can also be elicited by the occurrence of visual transients at locations different from that of the saccadic goal.

One might wonder what the mechanism underlying the saccadic boost observed in the present study may be. The onset of the peripheral stimuli might have caused an increment of discharge in those motor neurons programming a reflexive saccade toward the corresponding position, both at the SC level and the frontal eye field (FEF) level (Munoz \& Everling, 2004). However, the resulting reflexive saccade was successfully prevented because of the top-down control exerted by the FEF (Guitton, Buchtel, \& Douglas, 1985; Machado \& Rafal, 2004). Nevertheless, inhibitory signals might have been sent from saccade-related neurons to fixation neurons, thus reducing their firing rate. Indeed, evidence exists that the balance of activation between motor neurons and fixation neurons controls saccade initiation (Dorris \& Munoz, 1998). Hence, a reduced activity of fixation neurons in the SC and FEF might have resulted in reduced saccadic latency toward the target.

Finally, one might speculate that the double-signal condition may have caused greater visual activity in the SC and FEF than did the single-signal condition, and may have improved neural synchronizations within and between the two structures (Sommer \& Wurtz, 1998). This, in turn, could have resulted in an increased and/or more efficient recruitment of motor units, which caused a higher saccadic peak velocity.

\section{REFERENCES}

ARndt, P. A., \& Colonius, H. (2003). Two stages in crossmodal saccadic integration: Evidence from a visual-auditory focused attention task. Experimental Brain Research, 150, 417-426.

Colonius, H., \& ARndT, P. (2001). A two-stage model for visualauditory interaction in saccadic latencies. Perception \& Psychophysics, 63, 126-147.

Corballis, M. C. (1998). Interhemispheric neural summation in the absence of the corpus callosum. Brain, 121, 1795-1807.

Dodds, C., Machado, L., Rafal, R., \& Ro, T. (2002). A temporal/ nasal asymmetry for blindsight in a localisation task: Evidence for extrageniculate mediation. NeuroReport, 13, 655-658.

Dorris, M. C., \& MunOz, D. P. (1998). Saccadic probability influences motor preparation signals and time to saccadic initiation. Journal of Neuroscience, 18, 7015-7026.
Dorris, M. C., Paré, M., \& Munoz, D. P. (1997). Neuronal activity in monkey superior colliculus related to the initiation of saccadic eye movements. Journal of Neuroscience, 17, 8566-8579.

Edelman, J. A., \& GoldberG, M. E. (2001). Dependence of saccaderelated activity in the primate superior colliculus on visual target presence. Journal of Neurophysiology, 86, 676-691.

Findlay, J. M. (1997). Saccade target selection during visual search. Vision Research, 37, 617-631.

Giray, M., \& Ulrich, R. (1993). Motor coactivation revealed by response force in divided and focused attention. Journal of Experimental Psychology: Human Perception \& Performance, 19, 131-153.

Godisn, R., \& Theeuwes, J. (2002). Programming of endogenous and exogenous saccades: Evidence for a competitive integration model. Journal of Experimental Psychology: Human Perception \& Performance, 28, 1039-1054.

Guitton, D., Buchtel, H. A., \& Douglas, R. M. (1985). Frontal lobe lesions in man cause difficulties in suppressing reflexive glances and in generating goal-directed saccades. Experimental Brain Research, 58, 1278-1291.

Hughes, H. C., Reuter-Lorenz, P. A., Nozawa, G., \& Fendrich, R. (1994). Visual-auditory interactions in sensorimotor processing: Saccades versus manual responses. Journal of Experimental Psychology: Human Perception \& Performance, 20, 131-153.

Itaya, S. K., \& Van Hoesen, G. W. (1983). Retinal projections to the inferior and medial pulvinar nuclei in the Old-World monkey. Brain Research, 269, 223-230.

Kramer, A. F., Cassavaugh, N. D., Irwin, D. E., \& Peterson, M. S. (2001). Influence of single and multiple onset distractors on visual search for singleton targets. Perception \& Psychophysics, 63, 952968.

Machado, L., \& Rafal, R. D. (2004). Control of fixation and saccades during an anti-saccade task: An investigation in humans with chronic lesions of oculomotor cortex. Experimental Brain Research, 156, 5563.

Miller, J. (1982). Divided attention: Evidence for coactivation with redundant signals. Cognitive Psychology, 14, 247-279.

Miller, J. (1986). Timecourse of coactivation in bimodal divided attention. Perception \& Psychophysics, 40, 331-343.

Miller, J. (2004). Exaggerated redundancy gain in the split brain: A hemispheric coactivation account. Cognitive Psychology, 49, 118154.

Miller, J., \& Reynolds, A. (2003). The locus of redundant-targets and nontargets effects: Evidence from the psychological refractory period paradigm. Journal of Experimental Psychology: Human Perception \& Performance, 29, 1126-1142.

MordKoff, J. T., \& YANTIS, S. (1991). An interactive race model of divided attention. Journal of Experimental Psychology: Human Perception \& Performance, 17, 520-538.

Munoz, D. P., \& Everling, S. (2004). Look away: The anti-saccade task and the voluntary control of eye movement. Nature Reviews Neuroscience, $\mathbf{5}, 218-228$.

RAAB, D. (1962). Statistical facilitation of reaction time: Energy summation or preparation enhancement? Psychological Review, 80, 489509.

Ramchandran, R. S., Manoach, D. S., Cherkasova, M. V., LindGren, K. A., Goff, D. C., \& Barton, J. J. S. (2004). The relationship of saccadic peak velocity to latency: Evidence for a new prosaccadic abnormality in schizophrenia. Experimental Brain Research, 159, 99107.

Sommer, M. A., \& Wurtz, R. H. (1998). Frontal eye field neurons orthodromically activated from the superior colliculus. Journal of $\mathrm{Neu}$ rophysiology, 80, 3331-3335.

ToDD, J. W. (1912). Reaction to multiple stimuli. New York: Science Press.

Walker, R., Deubel, H., Schneider, W. X., \& Findlay, J. M. (1997). Effect of remote distractors on saccade programming: Evidence for an extended fixation zone. Journal of Neurophysiology, 78, 1108-1119.

(Manuscript received July 15, 2005; revision accepted for publication March 24, 2006.) 